\title{
Renal collision tumour of papillary cell carcinoma and chromophobe cell carcinoma with sarcomatoid transformation: A case report and review of the literature
}

\author{
Zhiqiang Zhang, MM; Jie Min, MD; Dexin Yu, MD; Haoqiang Shi, MD: Dongdong Xie, MD
}

Department of Urology, the Second Affiliated Hospital of Anhui Medical University, Anhui Province, China

Zhang Zhiqiang and Min Jie have equally contributed to this article.

Cite as: Can Urol Assoc J 2014;8(7-8):e536-9. http://dx.doi.org/10.5489/cuaj.1811

Published online August 11, 2014.

\section{Abstract}

The simultaneous occurrence of different histological types of adjacent neoplasms in the same organ is called a collision tumour, and rarely occurs in the kidney. A 63-year-old female presented to the urology department with a 1-month history of a painless hematuria. The computed tomography scans of the abdomen revealed 2 heterogeneous incidental right renal masses. The patient underwent radical nephrectomy including lymphadenectomy without adjuvant therapy. Histopathological examination showed a type 2 renal collision tumour of the papillary renal cell carcinoma with eosinophilic cytoplasm and pseudostratified nuclei on papillary cores, and chromophobe renal cell carcinoma characterized by large polygonal cells with transparent reticulated cytoplasm and prominent cell membranes, which exhibited sarcomatoid transformation in the local area. Routine follow-up demonstrated no local or distant metastasis signs of recurrence at 20 months.

\section{Introduction}

A collision tumour is characterized by the coexistence of two adjacent but histologically distinct malignant tumours in the same organ with no histological admixture. ${ }^{1.2}$ These kinds of tumours are rare. We present an extremely rare case of a renal collision tumour of the papillary cell carcinoma and chromophobe cell carcinoma, and with sarcomatoid transformation; to our knowledge, this is the first reported case..$^{2-4}$

\section{Case report}

A 63-year-old female presented to the urology department with a 1-month history of a painless hematuria without history of fever, weight loss, fatigue or urinary symptoms. Physical examination showed that her vital signs were stable and no superficial lymph nodes were found. Results from an electrocardiogram, pulmonary function test, stool analysis and other routine laboratory tests were all within normal limits. A voided urine cytology, however, revealed nonmalignant cells. Vomputed tomography (CT) scans of the abdomen also revealed two heterogeneous incidental right renal masses (Fig. 1, part A): a 4.5-cm solid, enhancing mass located in the anterior aspect of the upper pole and a 2.5-cm mass located along the mid lateral aspect of the kidney (Fig. 1, part B).

The patient underwent radical nephrectomy including lymphadenectomy without adjuvant therapy. The cut surface of the resected specimen showed two distinct tumours. The larger, light-brown and yellowish solid tumour, centred in the upper pole of kidney measured $5.0 \times 3.5 \times 3.0 \mathrm{~cm}$, while the second yellowish tumour, located in the middle portion that originated from the medulla but mostly extended to the renal pelvis, measured $3.0 \times 2.5 \times 2.5 \mathrm{~cm}$. Histologically, the larger tumour exhibited the features of papillary cell carcinoma, comprising papillary and tubulopapillary structures covered by higher nuclear grade with eosinophilic cytoplasm and pseudostratified nuclei on papillary cores (Fig. 2, part A). The second tumour exhibited the features of chromophobe cell carcinoma, characterized by large polygonal cells with transparent slightly reticulated cytoplasm and prominent cell membranes, and with sarcomatoid transformation in some areas (Fig. 2, part B).

Routine follow-up demonstrated no local or distant metastasis signs of recurrence at 20 months.

\section{Discussion}

A collision tumour is characterized by the coexistence of two adjacent but histologically distinct malignant tumours in the same organ with no histological admixture; ${ }^{1,2}$ if there is histological admixture of both tumours in the same organ, it is called a mixed tumour. Although collision tumours may appear in various organs, included the ovaries, colon, lung, stomach and skin, renal collision tumours are extremely rare. 

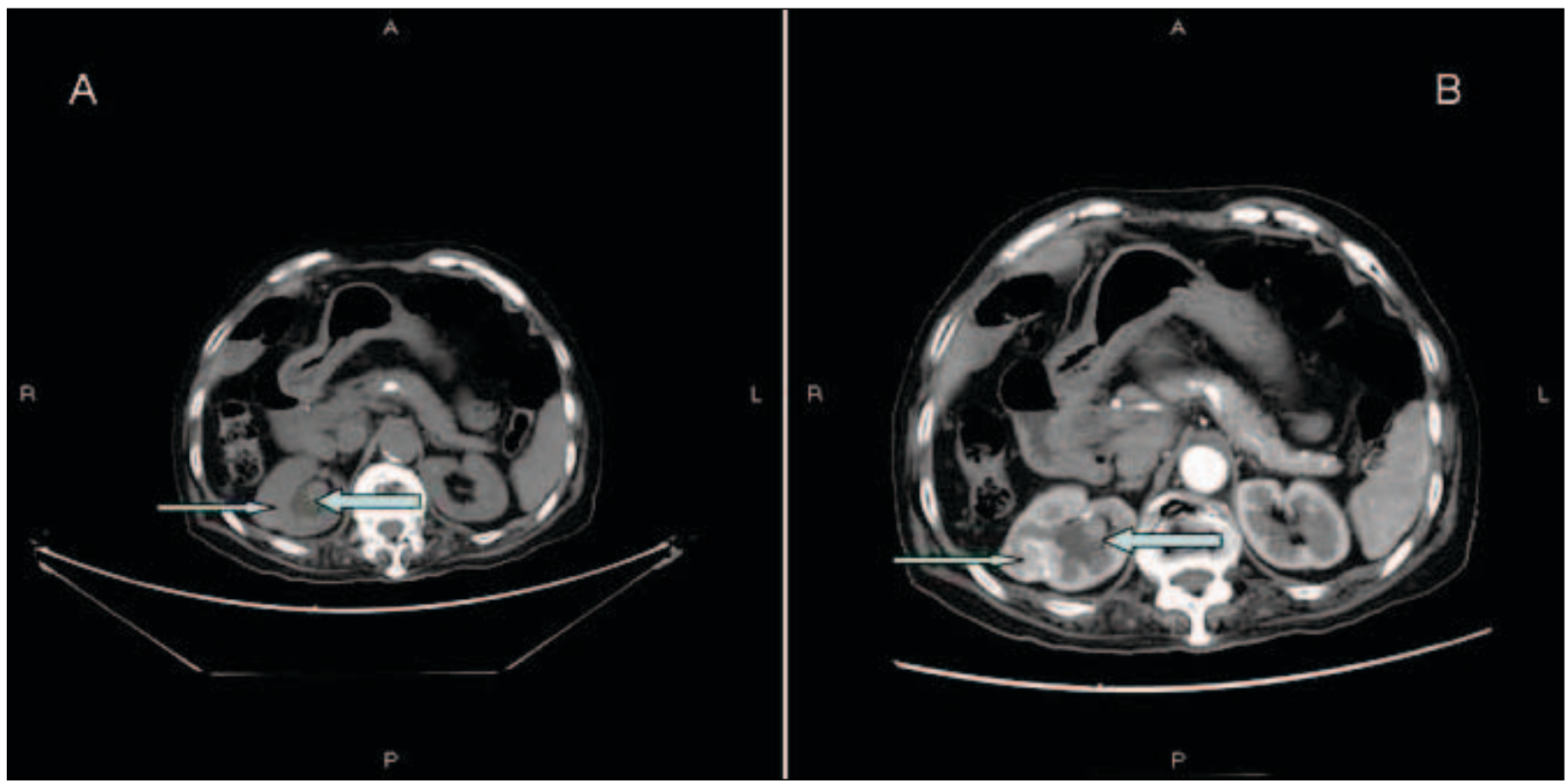

Fig. 1. The tumour is localized in the upper pole of right kidney and is heterogeneous with central lower density $(A)$ and attenuation with peripheral enhancement and central irregular enhancement (B). (The wide arrowhead points to ChRCC; the narrow arrow head points to PRCC.)

Valderrama and colleagues ${ }^{5}$ report a renal collision tumour comprising renal cell carcinoma (RCC) and squamous cell carcinoma (SCC), and a collision tumour consisting of chromophobe renal cell carcinoma (ChRCC) and collecting duct carcinomatous in a 64 year-old woman. ${ }^{6}$ In addition, PRCC and medullary carcinoma occurred in this tumour. ${ }^{7}$ On the basis of the pathological findings, the renal neoplasms was diagnosed as a collision tumour comprising papillary renal cell carcinoma (PRCC) and ChRCC with sarcomatoid transformation in the kidney; to our knowledge this is the first reported case..$^{2-4}$

RCC is a heterogeneous tumour that comprises different histological subtypes (based on morphology), including clear cell renal carcinoma, PRCC, ChRCC and collecting duct carcinomas. PRCC is the second-most common histological subtype, accounting for about $10 \%$ to $15 \%$ of all RCC. ${ }^{5,6}$ PRCC is characterized by malignant epithelial cells forming varying proportions of papillae and tubules and is subdivided into two morphologic groups, type 1 and type 2, based on cytologic and histologic features. Type 2 PRCC usually shows a higher stage and nuclear grade than type1 tumours ${ }^{8-10}$ and is associated with poorer survival rates. Immunohistochemistry can be helpful to confirm the diagnosis of PRCC, and show positivity in Cytokeratin7, CD10, AMACR and negativity for E-cadherin and CD117..$^{-11} \mathrm{In}$ rare instances, PRCC has been reported in renal collision tumours, but a collision tumour comprising PRCC and
ChRCC with sarcomatoid transformation has not been reported in the English literature to date.

ChRCC is a rare subtype of RCC first described by Thoenes et al. in $198 .{ }^{12}$ It represents only $5 \%$ to $10 \%$ of the total cases of $\mathrm{RCC}^{13} \mathrm{ChRCC}$ is a well-circumscribed solid mass with broad fibrotic septa. The surface of the mass is usually homogeneously beige, light tan, brown or yellow. ChRCC comprises large, polygonal pale cells with abundant transparent cytoplasm and prominent cell membranes, and small cells with granular and eosinophilic cytoplasm. ${ }^{8,14}$ It is divided into different subtypes, including classic, eosinophilic and mixed, based on the proportion of cells.

Immunohistochemistry shows positivity in cytokeratin 7, CD82, Parvalbumin and ksp-cadherin, and negativity for CD10 and CA9. ${ }^{14,15}$ Sarcomatoid dedifferentiation rarely occurs with any type of RCC, although it does commonly present in ChRCC. Sarcomatoid dedifferentiation is characterized by spindle-like cells, high cellularity and cellular atypia, commonly associated with necrosis and microvascular invasion. ${ }^{15,16}$ ChRCC has a better prognosis and a lower tendency to progress and metastasize than do other subtypes of RCC, but ChRCC with sarcomatoid change is characterized by its highly malignant biological behaviour, represented as an independent prognostic factor in ChRCC. ${ }^{15-18}$

There are differing hypotheses on the histogenesis of renal collision tumours. ${ }^{1}$ It is clearly an incidental phenomenon when more than two unrelated tumours occur in the same 


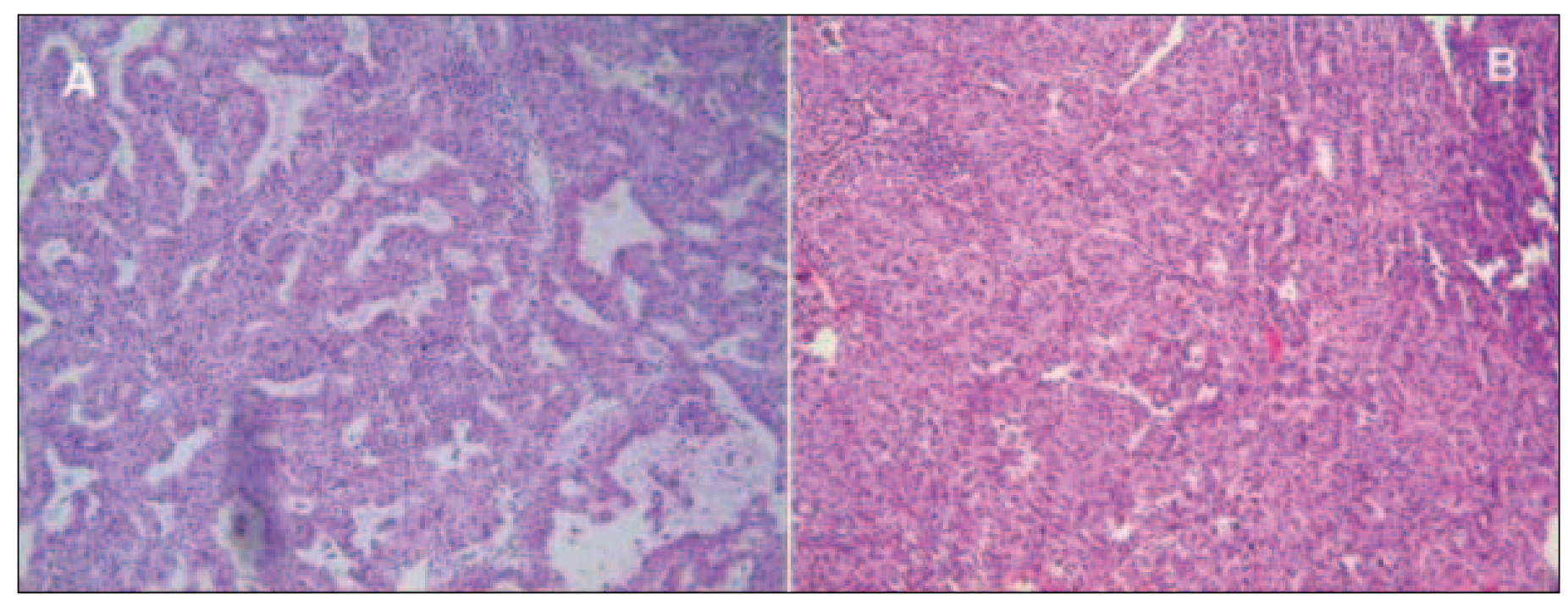

Fig. 2. Histopathological examination of the renal collision tumour (HE, original magnification $\times 100)$. Type 2 PRCC tumour cells are of higher nuclear grade with eosinophilic cytoplasm and pseudostratified nuclei on papillary cores (A). ChRCC is cytoplasm and prominent cell membranes, and shows sarcomatoid transformation in the local area (B).

organ at the same time. ${ }^{2}$ One hypothesis centres around the cell proliferation theory: although PRCC and ChRCC originate from renal parenchymal, they arise from different cells, and a collision tumour occurs because of the simultaneous occurrence of both different kinds of cell proliferation and/ or differentiation. ${ }^{3}$ Another hypothesis centres around the cancer stem cell theory, in which the occurrence of collision tumours may be related to the ability of cancer stem cells to differentiate into different tumour cell lines in the same organ or anatomic site. ${ }^{19}$ Of course, both of these theories may be rational interpretations of the collision tumour phenomenon.

In this case, the diagnosis of renal collision tumour of PRCC and ChRCC with sarcomatoid transformation was confirmed on microscopic morphological features and immunohistochemistry, and the differential diagnosis included clear cell RCC and renal pelvic cancer. In addition, a collision tumour of the kidney should be distinguished from a composite tumour within which different tumour histologic components coexist. In this case, histopathological examination showed a type 2 PRCC renal collision tumour with eosinophilic cytoplasm and pseudostratified nuclei on papillary cores, and ChRCC characterized by large polygonal cells with transparent reticulated cytoplasm and prominent cells that exhibited sarcomatoid transformation in the local area. Renal collision comprises distinct benign and/or malignant areas that sometimes may not appear on a biopsy because the text may not sufficiently evaluate the tumour's components. ${ }^{2}$ Pathologists must therefore pay rigorous attention to the gross examination to find the malignant areas which offer evidence for therapy and prognosis.

Although there is no standard treatment for renal collision tumour at present, complete surgical excision of the lesion may be the only potentially curative treatment. There is little available information on a large series with long-term follow-up; as well, the therapy and prognosis of renal collision tumours have not been properly clarified. To our knowledge, the different subtypes of renal tumours have varying diseasefree survival rates after treatment; in particular, ChRCC has a better outcome. ${ }^{18,20}$ For this reason, histological subtypes represent an independent prognostic factor of renal collision tumours, so the timing and frequency of follow-up should be based on the different subtypes. Current studies confirm that patients with ChRCC who are treated with targeted agents (sorafenib and sunitinib) achieve partial response, ${ }^{21,22}$ but a few reports on RCC show sarcomatoid change using targeted therapies. In addition, ChRCC with sarcomatoid change tend to respond to chemotherapy, including gemcitabine and doxorubicin.

This patient underwent right nephrectomy with sorafenib. Routine follow-up demonstrated no local or distant metastasis signs of reccurrence at 20 months. Until additional data are available, clinicians should consider treating ChRCC with sarcomatoid dedifferentiation with sorafenib.

\section{Conclusion}

We have described two adjacent but histologically distinct malignant tumours occurring in the kidney without histological admixture. These renal collision tumours comprised papillary cell carcinoma and chromophobe cell carcinoma and showed sarcomatoid transformation, which is exceedingly rare.

Complete surgical excision may be the only potentially curative treatment, and sorafenib is a better choice of 
adjunctive therapy. The few existing published case reports may not be sufficient to allow the clinical outcome to be fully evaluated, however, so a longer follow-up period may be necessary to definitively evaluate the clinical outcome of renal collision.

Competing interests: Dr. Zhang, Dr. Min, Dr. Yu, Dr. Shi and D. Xie all declare no competing financial or personal interests.

This paper has been peer-reviewed.

\section{References}

1. Rabbani F, Grimaldi G, Russo P. Multiple primary malignancies in renal cell carcinomas. J Urol 1998;160:1255-59

2. Seiben I, Szabó Z, Lukács N, et al.Papillary renal cell carcinoma embedded in an oncocytoma: Case report of a rare combined tumour of the kidney. Can Urol Assoc J 2013;7:7-8.

3. Floyd MS Jr, Javed S, Pradeep KE,et al. Composite oncocytoma and papillary renal cell carcinoma of the kidney treated by partial nephrectomy: A case report. Scientific World Journal 2011;11:1173-77.

4. Rowsell C, Fleshner N, Marrano P, et al. Papillary renal cell carcinoma within a renal oncocytoma: Case report of an incidental finding of a tumour within a tumour. J Clin Pathol 2007;60:426-8.

5. Valderrama E, Kalra J, Badlani $G$, et al. Simultaneous renal cell carcinoma and squamous cell carcinoma of kidney. Urology 198;29:441-5.

6. Kawano N, Inayama Y, Nakaigawa N, et al. Composite distal nephron-derived renal cell carcinoma with chromophobe and collecting duct carcinomatous elements. Pathol Int 2005;55:360-365.

7. Lam ET, Kessler ER, Flaig TW, et al. Collision renal cell papillary and medullary carcinoma in a 66-year-old man. Oncology (Williston Park) 2013;27:893, 896, 898.

8. Lopez-Beltran A, Scarpelli M, Montironi R, et al. 2004 WHO classification of the renal tumours of the adults. Eur Urol 2006;49:798-805.

9. Delahunt B, Eble JN, McCredie MR, Bethwaite PB,Stewart JH, Bilous AM. Morphologic typing of papillary renal cell carcinoma: Comparison of growth kinetics and patient survival in 66 cases. Hum Pathol 2001;32:590-5
10. Al-Ghawi H, Asoyo OA, Truong LD, et al. Application of immunohistochemistry to the diagnosis of kidney tumours. Pathol Case Rev 2010;15:25-34.

11. Truong LD, Shen SS. Immunohistochemical diagnosis of renal neoplasm. Arch Pathol Lab Med 2011;135:92-109.

12. Thoenes W, Storkel S, Rumpelt HJ. Human chromophobe cell renal carcinoma. Virchows Arch. B Cell Pathol. Incl. Mol. Pathol. 1985:48:207-17.

13. Siegel R, Ward E, Brawley 0, Jemal A. Cancer statistics, 2011: The impact of eliminating socioeconomic and racial disparities on premature cancer deaths. CA Cancer J. Clin 2011;61:212-36.

14. Vera-Badillo FE, Conde E, Duran I. Chromophobe renal cell carcinoma: A review of an uncommon entity. Int I Urol 2012;19:894-900.

15. Amin MB, Paner GP, Alvarado-Cabrero I, et al. Chromophobe renal cell carcinoma: Histomorphologic characteristics and evaluation of conventional pathologic prognostic parameters in 145 cases. Am I Surg Pathol 2008;32:1822-34.

16. Kuroda N, Tanaka A, Ohe C, Nagashima Y. Recent advances of immunohistochemistry for diagnosis of renal tumours. Pathol Int 2013;63:381-90.

17. Vera-Badillo FE, Conde E, Duran I. Chromophobe renal cell carcinoma: A review of an uncommon entity. Int J Urol 2012:19:894-900.

18. Kuroda N, Tanaka A.Recent classification of renal epithelial tumours. Med Mol Morphol 2013 Mar 26. [Epub ahead of print]

19. Izawa $\mathrm{T}$, Kuwamura $M, 0$ kamura $\mathrm{K}$, et al. A collision tumour consisting of granular cell tumour and adenocarcinoma in the uterus of an aged djungarian hamster. J Toxicol Pathol 201 1;24:233-7.

20. Volpe A, Novara G, Antonelli A, et al. Martignoni G, Minervini A, Mirone V, Simonato A, Terrone C, Zattoni F,Ficarra V. Chromophobe renal cell carcinoma (RCC): Oncological outcomes and prognostic factors in a large multicentre series. BJU Int.2012;110:76-83.

21. Choueiri TK, Plantade A, Elson P, et al. Efficacy of sunitinib and sorafenib in metastatic papillary and chromophobe renal cell carcinoma. J Clin Oncol 2008;26:127-31.

22. Staehler M, Haseke N, Roosen A, et al. Sorafenib after combination therapy with gemcitabine plus doxorubicine in patients with sarcomatoid renal cell carcinoma: A prospective evaluation. Eur J Med Res 2010;15:287-91.

Correspondence: Yu Dexin, Department of Urology, The Second Affiliated Hospital of Anhui Medical University; zhangzq_urology@163.com 